\title{
活性氧响应的新型阳离子共聚物构建及其基因递送性能研究
}

\author{
韩旭张留伟张强睢晞航钱明陈麒先* 王静云* \\ (大连理工大学 生物工程学院 大连 116024)
}

\begin{abstract}
摘要 引入快速、主动释放基因的机制是提升非病毒基因递送效率的关键. 本研究以 2-甲基丙烯酰氧乙基磷酰胆碱 (MPC)与(2-丙烯酰基)乙基(硼酸芐基)二乙基澳化铵(BD)为单体, 基于可逆加成-断裂链转移聚合(RAFT)反应制备具有 活性氧响应性质的聚阳离子嵌段共聚物 $\mathrm{pM}$-pBD. 通过静电作用, 阳离子共聚物 pM-pBD 能够与带负电荷的 DNA 以分 子自组装的方式形成纳米复合物. 其中, 阳离子 $\mathrm{pBD}$ 片段具有活性氧触发电荷反转的特性, 因此, 有助于获得活性氧 触发的静电组装复合体结构解离, 从而实现可控基因释放的功能. 理化表征结果表明, pM-pBD 与质粒 DNA 静电复合 后能够形成粒径约为 $99.1 \mathrm{~nm}, \zeta$ 电位约为 $+13.8 \mathrm{mV}$, 显微形貌近似球形的纳米复合物. 在加入促过氧化氢产生的抗坏 血酸后，上述 $\mathrm{pM}-\mathrm{pBD}$ 基因递送系统的转染效率得到了显著的提升. 因此，本研究创制的 $\mathrm{pM}-\mathrm{pBD}$ 为基因递送系统的可 控释放提供了新的解决方案.
\end{abstract}

关键词＼cjkstart聚阳离子；电荷反转；基因递送；活性氧响应；抗坏血酸

\section{Construction of a Novel Reactive Oxygen Species-responsive Cationic Copolymer and Its Performance in Gene Delivery}

\author{
Xu Han Liuwei Zhang Qiang Zhang Xihang Sui Ming Qian \\ Qixian Chen* Jingyun Wang* \\ (School of Bioengineering, Dalian University of Technology, Dalian 116024, China)
}

\begin{abstract}
The development of rapid and active gene release function is the key to improve the efficiency of non-viral gene delivery system. Herein, a novel reactive oxygen-responsive cationic block copolymer non-viral gene delivery vector (termed as pM-pBD) consisting of biocompatible poly(2-methacryloyloxyethyl phosphorylcholine) (pMPC) segment and charge reversible poly[(2-acryloyl)-ethyl-(boronic acid benzyl)-diethylammonium bromide] segment (pBD) was synthesized via reversible addition-fragmentation chain transfer polymerization (RAFT). Dynamic light scattering (DLS) assay and $\zeta$ potential measurements, transmission electron microscope (TEM) and gel electrophoresis were performed to characterize pM-pBD/(plasmid DNA, pDNA) complexes. Gel electrophoresis retardation assay displayed that pM-pBD can firmly bind pDNA through electrostatic interaction even in the presence of high concentration of heparin, but capable of releasing pDNA in response to reactive oxygen (such as $\mathrm{H}_{2} \mathrm{O}_{2}$ ). The pM-pBD/pDNA complexes show low cytotoxicity against HeLa cells, even at high weight ratio of pM-pBD and pDNA (N/P) (up to 16) demonstrated by MTT (methylthiazolyldiphenyltetrazolium bromide) assay. The $\mathrm{pM}-\mathrm{pBD} / \mathrm{pDNA}$ complexes demonstrated appreciable colloidal stability in the presence of $10 \%$ fetal bovine serum. The $\mathrm{pM}-\mathrm{pBD} / \mathrm{pDNA}$ complexes at N/P ratio of 3 displayed spherical morphologies with average diameter of approximate $99.1 \mathrm{~nm}$ and $\zeta$ potential of approximate $+13.8 \mathrm{mV}$. However, once upon incubation in presence of $1 \mathrm{mmol} / \mathrm{L} \mathrm{H}_{2} \mathrm{O}_{2}$, the diameter of $\mathrm{pM}-\mathrm{pBD} / \mathrm{pDNA}$ complexes at $\mathrm{N} / \mathrm{P}$ ratio of 3 was enlarged to $330 \mathrm{~nm}$ and $\zeta$ potential was reversed to $-3.91 \mathrm{mV}$ due to charge reversal of $\mathrm{BD}$ responsive to reactive oxygen. Flow cytometry revealed uptake efficiency (88.9\%) and the highest transfection efficiency (31.2\%) for pM-pBD at N/P ratio of 3 and 16, respectively. The transfection efficiency of pM-pBD/pDNA against HeLa cells was observed to be significantly augmented (1.5-fold) after the addition of ascorbic acid, which could stimulate the production of hydrogen peroxide. Therefore, pM-pBD represented intriguing utilities in fabrication of non-viral gene delivery systems, which enable spatiotemporal control of gene release and thereby facilitated the subsequent transcription machinery.

Keywords polycation; charge reversal; gene delivery; ROS-responsive; ascorbic acid
\end{abstract}

\footnotetext{
*E-mail: qixian@dlut.edu.cn; wangjingyun67@dlut.edu.cn; Tel.: 0411-84706805

Received March 11, 2021; published April 30, 2021.

Supporting information for this article is available free of charge via the Internet at http://sioc-journal.cn.

Project supported by the National Natural Science Foundation of China (Nos. 21878041, 22078050), the Fundamental Research Funds for the Central Universities (Nos. DUT17RC(3)059, DUT20YG126), and Dalian Science \& Technology Innovation Fund (2020JJ26SN050, 2020JJ26GX025).

项目受国家自然科学基金(Nos. 21878041, 22078050)、中央高校基本科研业务费专项资金(Nos. DUT17RC(3)059, DUT20YG126)和大连市科技创新基 金(Nos. 2020JJ26SN050, 2020JJ26GX025)资助.
} 


\section{1 引言}

基因治疗是指将外源基因导入靶细胞内，以取代、 补偿或者纠正异常基因, 以达到疾病治疗的目的 ${ }^{[1-2]}$. 基 因治疗的概念在 1972 年被首次提出, 自此以后, 基因疗 法飞速发展. 目前, 基因治疗已经应用于多种疾病的治 疗 ${ }^{[3-7]}$. 但基因治疗在临床上大规模应用仍然受到缺乏 高效、安全的基因递送系统的限制. 病毒载体是最早开 发出来的基因治疗递送系统, 在基因治疗研究的前期占 主要地位, 但其发展因基因装载率低、成本高、缺乏特 异性和靶向性以及不可预测的安全性等问题而在临床 应用中受到限制 $[8-11]$. 因此, 开发高效、安全的非病毒载 体一直是基因递送领域的研究热点.

非病毒载体主要包括脂质体、阳离子共聚物以及树 枝状大分子等 ${ }^{[12]}$. 其中, 阳离子共聚物能与带负电荷的 细胞膜相结合, 利于内吞, 进入胞内后能促进溶酶体逃 逸, 从而提高载体的基因转染效率 ${ }^{[13]}$. Zhou 等 ${ }^{[14]}$ 将 $N, N$ 二甲基氨乙基甲基丙烯酸酯与甲基丙烯酸羟基乙酯通 过 RAFT (reversible addition-fragmentation chain transfer polymerization)的方法合成和制备了粒径为 $100 \mathrm{~nm} 、 \zeta$ 电位为 $+40 \mathrm{mV}$ 的阳离子聚合物载体用来递送基因, 但 是没有明确的释放机制, 只能依靠胞内物质竞争缓慢释 放, 转染效率不高. 目前报道的阳离子共聚物释放基因 的机制大多是缓慢且被动的, 因此设计智能响应, 主动 释放基因的阳离子共聚物基因载体, 对提高基因转染效 率具有重要意义.

活性氧(ROS), 如单线态氧、超氧阴离子、羟基自 由基及过氧化氢等, 在许多生理过程中起着关键性作 用 ${ }^{[15-16]}$. 据报道, 正常组织细胞中 ROS 浓度在 $0.5 \sim 7.0$ $\mu \mathrm{mol} / \mathrm{L}$ 之间, 而肿瘤组织细胞中的 ROS 浓度可以达到 $1.0 \mathrm{mmol} / \mathrm{L}^{[17-18]}$. Zhang 等 ${ }^{[19]}$ 通过二环氧开环聚合的方 法设计并制备了一系列氟苯取代的具有 ROS 反应性硫
缩醛键的聚阳离子(TAEA-S-xF), 研究结果表明, 随着 氟原子数目的增加, 这些聚阳离子具有较好的转染效率 和抗血清性，有助于克服传递过程中的胞内屏障. Shim 等 ${ }^{[20]}$ 将寡聚胺与丙烯酰胺硫缩酮交联剂聚合, 合成了 ROS 响应型阳离子水溶性共聚物, 该共聚物被证明在 癌细胞中能够有效安全地传递基因, 癌症靶向肽的修饰 能够避免正常细胞中的非特异性积累, 并且提高基因的 传递效率, 从而降低潜在的全身毒性. 上述阳离子聚合 物虽然能够快速、主动地释放基因, 但是仍然存在生物 相容性低、细胞毒性高及转染效率低等缺点.

本研究旨在构建一种新型活性氧响应的阳离子共 聚物基因载体 $\mathrm{pM}-\mathrm{pBD}$, 该载体能够响应活性氧而发生 电荷反转进而释放出所载基因(图式 1). 本文研究了该 新型载体结合 DNA 的能力, 同时评价其细胞毒性和作 为智能释放型基因载体对含有不同 ROS 水平 HeLa 细胞 的转染效率.

\section{2 结果与讨论}

\section{1 阳离子共聚物(pM-pBD)的合成及结构表征}

按照图 1 所示的合成路线合成聚阳离子共聚物 $\mathrm{pM}-\mathrm{pBD}$. 首先通过丙烯酸 $N, N$-二乙基氨基乙酯 (DEAEA)和 4-澳甲基苯硼酸频哪醇酯(B)在二甲基甲酰 胺中反应合成了带有阳离子的(2-丙烯酰)-乙基-(硼酸茮 基)-二乙基溴化铵单体(BD). 然后通过 RAFT 法合成了 聚(2-甲基丙烯酰氧乙基磷酰胆碱)(pMPC), pMPC 可以 作为下一步聚合反应的链转移剂, 继续将 $\mathrm{BD}$ 聚合到 pMPC 主链上, 经过透析除杂、真空干燥后获得活性氧 响应型阳离子共聚物分子 $\mathrm{pM}-\mathrm{pBD}$. 所合成的产物和中 间体通过核磁共振氢谱及高分辨质谱证明结构正确(图 $\mathrm{S} 1 \sim \mathrm{S} 4)$. 通过对核磁共振氢谱的分析计算可知，阳离 子共聚物中 MPC 的聚合度约为 $39, \mathrm{BD}$ 的聚合度约为
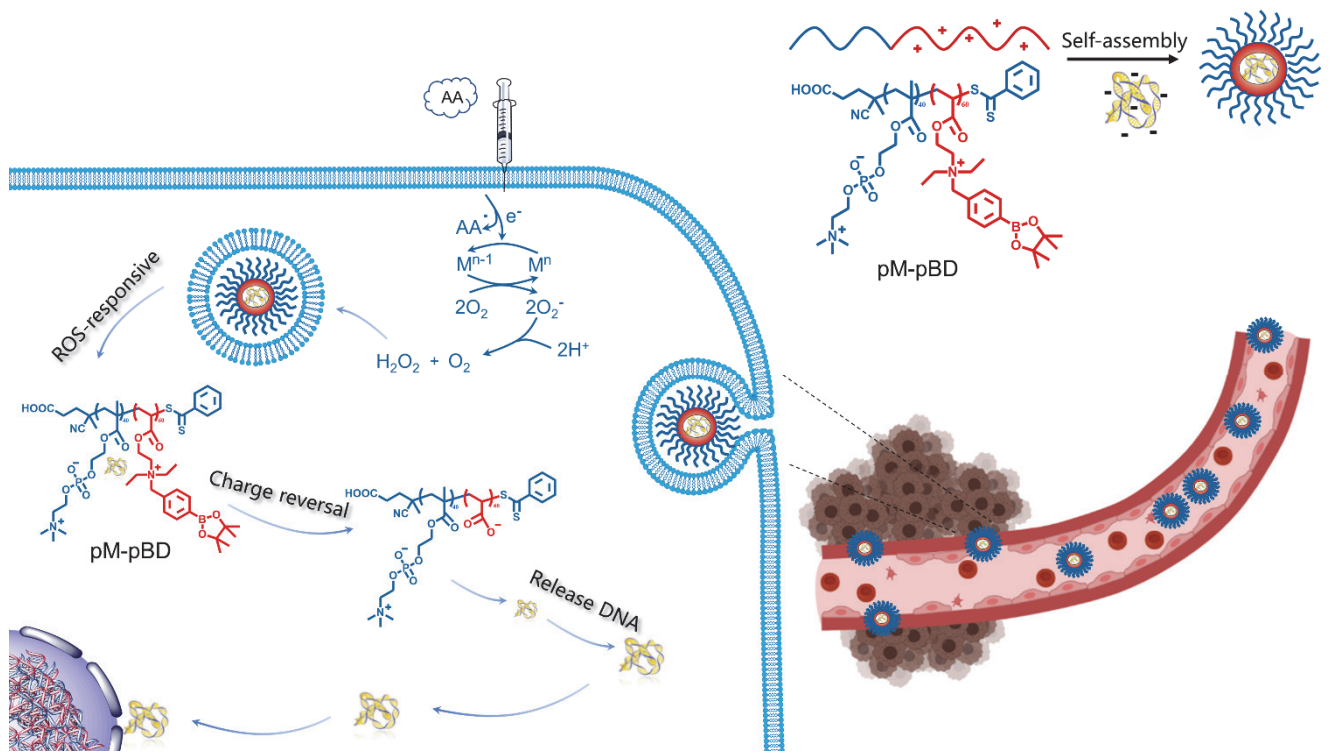

图式 1 载体与基因自组装形成纳米复合物及细胞内响应活性氧实现电荷反转释放 DNA 过程示意图

Scheme 1 Schematic representation of non-viral gene delivery system from reaction oxygen species (ROS)-responsive charge-reversal copolymers 


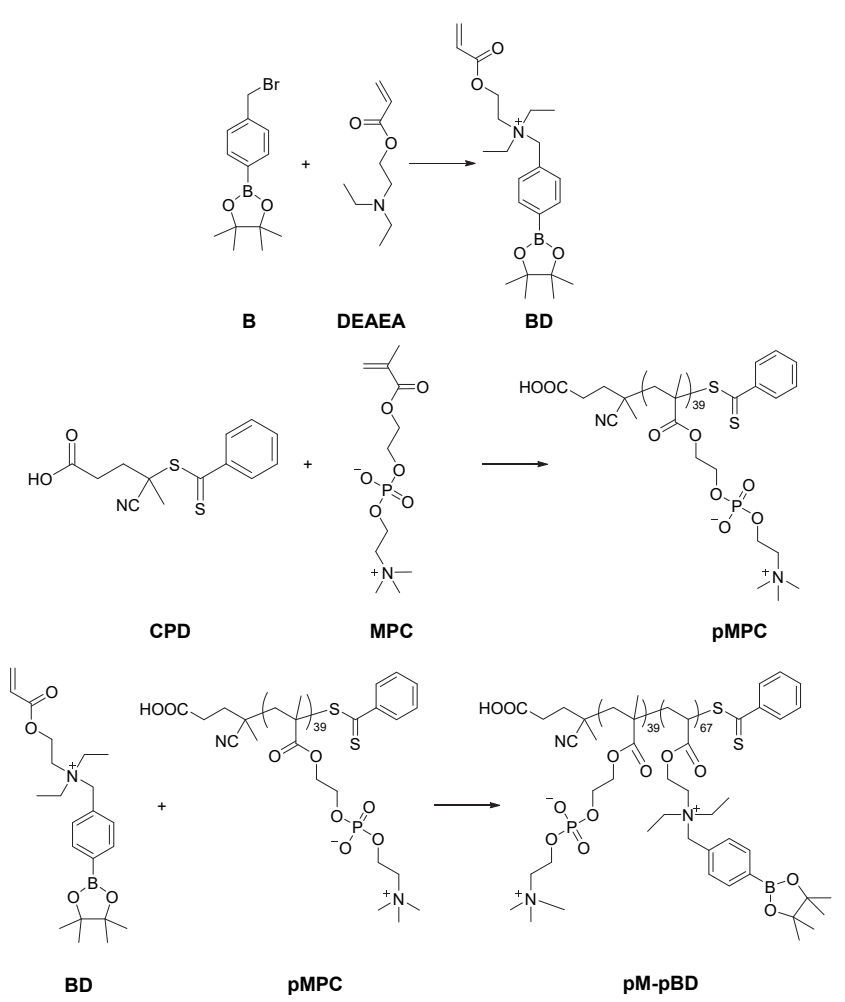

图 1 活性氧响应的新型阳离子共聚物 $\mathrm{pM}-\mathrm{pBD}$ 的合成路线

Figure 1 Synthetic route of ROS-responsive cationic copolymeric pM-pBD

(a)

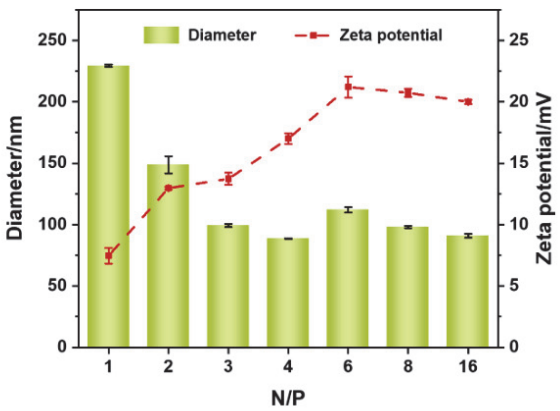

(c)

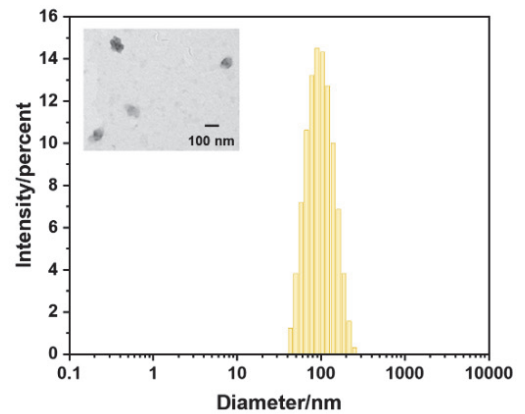

67, 计算出 pM-pBD 的分子量约为 $37812 \mathrm{Da}$. 此外, 凝 胶渗透色谱(gel permeation chromatography, GPC)测定 结果显示 $\mathrm{pM}-\mathrm{pBD}$ 分子的 $M_{\mathrm{n}}$ 为 $38338 \mathrm{Da}, M_{\mathrm{w}}$ 为 42138 $\mathrm{Da}, \mathrm{PDI}$ 为 1.10 (图 S5).

\section{$2.2 \mathrm{pM}-\mathrm{pBD} / \mathrm{pDNA}$ 纳米复合物的制备及表征}

为了验证带有正电荷的 $\mathrm{pM}-\mathrm{pBD}$ 与质粒 DNA (pDNA)通过静电作用进行自组装的行为, 首先测定了 形成的纳米复合物的粒径和表面电位. 如图 $2 \mathrm{a}$ 所示, 在 不同 N/P (N/P 为载体 $\mathrm{pM}-\mathrm{pBD}$ 与 $\mathrm{pDNA}$ 的质量比, N/P: $1 \sim 16)$ 下, 纳米复合物的粒径在 $88 \sim 230 \mathrm{~nm}$ 之间, 电位 在 $+6 \sim+30 \mathrm{mV}$ 之间. 随着 $\mathrm{N} / \mathrm{P}$ 的升高, 纳米复合物的 粒径逐渐变小, 电位逐渐上升. 这是由于带正电荷的载 体越多，压缩 DNA 的能力增强，使得形成的纳米复合 物的粒径越小. 随后, 利用 DLS 测定了纳米复合物在含 有血清的缓冲液中的粒径变化. 结果如图 $2 \mathrm{~b}$ 所示, 当 $\mathrm{N} / \mathrm{P}$ 值为 1,2 和 3 时, 纳米复合物在含有 $10 \%$ 血清的缓 冲液中粒子的粒径几乎没有变化, 说明此时的纳米复合 物具有良好的血清稳定性; 但是，在其他 N/P 值(4, 6, 8 和 16)时, 尤其是 $N / P \geqslant 6$ 的载体复合物的粒径随着孵育 时间的增加显著增大，可能是由于此时的复合物带有过 多的正电荷，易吸附血清中带有负电的蛋白质而发生才 聚. 结合以上实验结果, 本实验选择 $\mathrm{N} / \mathrm{P}=3$ 的纳米复合 物进行后续的实验.

(b)

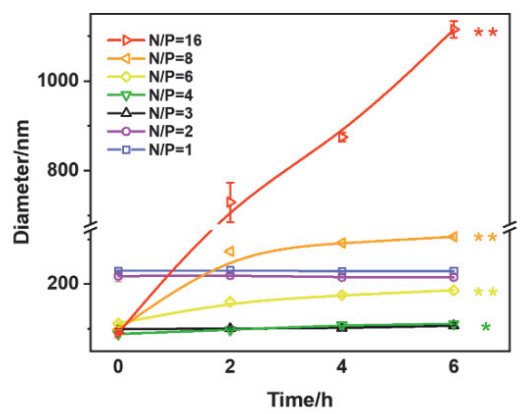

(d)

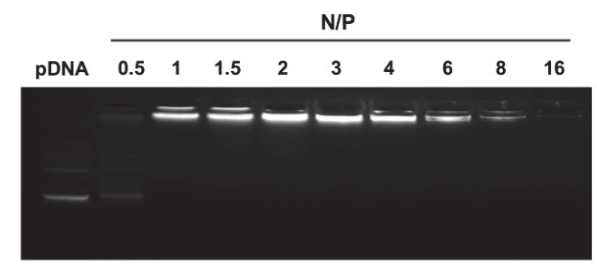

图 2 (a)不同 $\mathrm{N} / \mathrm{P}$ 纳米复合物的粒径和表面电位. (b)动态光散射法测定不同 $\mathrm{N} / \mathrm{P}$ 时, pM-pBD/pDNA 在含有 $10 \%$ 胎牛血清的缓冲液中粒径变化. (c) 在 $\mathrm{N} / \mathrm{P}=3$ 时 $\mathrm{pM}-\mathrm{pBD} / \mathrm{pDNA}$ 复合物的粒径分布和 $\mathrm{TEM}$ 显微图片(插图). (d)不同 N/P 时, pM-pBD/pDNA 的凝胶阻滞电泳图

Figure 2 (a) The particle size and $\zeta$ potential of $\mathrm{pM}-\mathrm{pBD} / \mathrm{pDNA}$ at varied N/P ratios. (b) The particle size of $\mathrm{pM}-\mathrm{pBD} / \mathrm{pDNA}$ in buffer containing $10 \%$ fetal bovine serum was measured by dynamic light scattering. (c) The particle size distribution and morphologies of $\mathrm{pM}-\mathrm{pBD} / \mathrm{pDNA}$ complex at $\mathrm{N} / \mathrm{P}=3$ by TEM (inset). (d) Gel electrophoresis image of pM-pBD/pDNA at varied N/P ratios 
当 $\mathrm{N} / \mathrm{P}=3$ 时, 纳米复合物的平均粒径约为 99.1 $\mathrm{nm}$ (图 2c), 且分布较窄, 透射电子显微镜(TEM)结果显 示 $\mathrm{pM}-\mathrm{pBD} / \mathrm{pDNA}$ 粒子呈现出球形或近似球形, 其大小 与动态光散射法(DLS)测定的结果一致. 一般来说, 粒 径在 $100 \mathrm{~nm}$ 左右的纳米颗粒能够有效地逃逸肝/肾清除 机制, 更高效地通过 EPR 效应(enhanced permeability and retention effect)到达病灶部位. 与此同时, 测定的 $\zeta$ 电位约为 $+13.8 \mathrm{mV}$, 带正电的纳米复合物能够通过静 电排斥在溶液中显示出较好的胶体稳定性, 而且能够与 带负电荷的细胞膜结合促进细胞内吞进而提高转染效 率. 此外, 通过凝胶阻滞实验验证了载体结合 DNA 的 能力, 结果如图 $2 \mathrm{~d}$ 所示, 当 $\mathrm{N} / \mathrm{P} \geqslant 1$ 时, DNA 被完全阻 滞在上样孔中, 表明在此条件下载体可以完全与 DNA 复合形成基因复合物. 而且, 随着 $\mathrm{N} / \mathrm{P}$ 的增加, 电泳条 带亮度逐渐减弱, 这可能是由于载体量的增加, DNA 被 高度凝缩并紧密地包裹在载体内核中. 最后, 利用肝素 竞争实验进一步研究了载体 pM-pBD 与 DNA 结合的能 力, 从结果(图 S6)可以看出, 随着肝素浓度的增加, 在 一定范围内, 基因载体复合物仍能够完全阻滞在上样孔 中, 说明了载体 $\mathrm{pM}-\mathrm{pBD}$ 与 DNA 具有较高的结合能力, 能够抵抗外界的干扰, 为长效的血液循环奠定了良好的 基础; 当肝素浓度高达 $20 \mathrm{mg} / \mathrm{mL}$ 时, 出现了与 $\mathrm{pDNA}$ 组对应的 DNA 条带, 说明 DNA 与载体复合后没有破坏 pDNA 结构. 综上所述, 载体 pM-pBD 能够与 DNA (N/P $=3$ )形成类似球形的纳米结构, 粒径在 $100 \mathrm{~nm}$ 左右, 并 且在溶液中具有良好的稳定性.

(a)

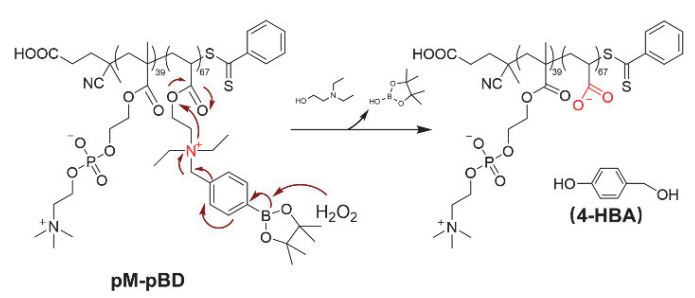

(c)
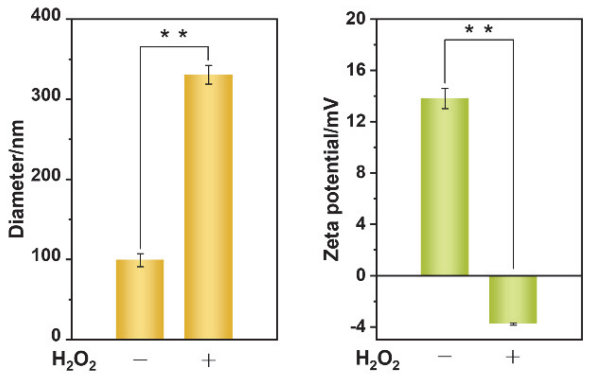

2.3 pM-pBD 载体及 $p M-p B D / p D N A$ 纳米复合物对 ROS 响应性分析

为了验证载体 pM-pBD 能够响应 ROS 发生级联断 裂反应，实现由正电荷到负电荷的电荷反转从而有利于 释放复合的 DNA(图 3a), 本文首先利用高效液相色谱法 ( $\mathrm{HPLC}$ )检测载体与 $\mathrm{H}_{2} \mathrm{O}_{2}$ 孵育后，释放到溶液中对羟基 苯甲醇(4-hydroxybenzyl alcohol, 4-HBA)的含量，结果 如图 $3 b$ 所示, 在 $1 \mathrm{mmol} / \mathrm{L} \mathrm{H}_{2} \mathrm{O}_{2}$ 孵育后, 随着时间的增 加, 在洗脱时间为 $6.5 \mathrm{~min}$ 处(4-HBA 吸收峰)出现了新的 吸收峰, 并逐渐增强, 说明了 $\mathrm{pM}-\mathrm{pBD}$ 在 $\mathrm{H}_{2} \mathrm{O}_{2}$ 作用下发 生级联断裂反应并逐渐释放 4-HBA. 相反, 在没有加入 $\mathrm{H}_{2} \mathrm{O}_{2}$ 时, 并未检测到 4-HBA 的吸收峰. 这些结果表明 了在 $\mathrm{H}_{2} \mathrm{O}_{2}$ 等 ROS 存在情况下, pM-pBD 能够发生级联 断裂反应脱去 4-HBA 形成带负电的羧基(图 3a), 为 ROS 刺激响应性电荷反转和载荷释放奠定了基础.

为了进一步研究载体的级联断裂会导致 $\mathrm{pM}-\mathrm{pBD} / \mathrm{pDNA}$ 纳米复合物电荷反转和释放载荷 (pDNA) 行为, 利用 DLS 测定了 $\mathrm{H}_{2} \mathrm{O}_{2}$ 孵育后的粒径和电 位. 如图 $3 \mathrm{c}$ 所示, $\mathrm{pM}-\mathrm{pBD} / \mathrm{pDNA}$ 纳米复合物在 1 $\mathrm{mmol} / \mathrm{L} \mathrm{H}_{2} \mathrm{O}_{2}$ 处理 $60 \mathrm{~min}$ 后, $\mathrm{pM}-\mathrm{pBD} / \mathrm{pDNA}$ 纳米复合 物粒径由原来的约 $99 \mathrm{~nm}$ 增大到约 $330 \mathrm{~nm}$, 说明了在氧 化环境中 $\mathrm{pM}-\mathrm{pBD} / \mathrm{pDNA}$ 复合物能够发生 ROS 刺激的 氧化分解反应，使得纳米复合物溶胀，结构变得松散. 此外, 表面电位由原来的约 $+13.8 \mathrm{mV}$ 降低到 -3.91 $\mathrm{mV}$, 这是由于 ROS 刺激后, $\mathrm{pM}-\mathrm{pBD}$ 脱去了带正电的季 铵基团变成了带负电的羧基, 实现了电荷反转功能, 并

(b)

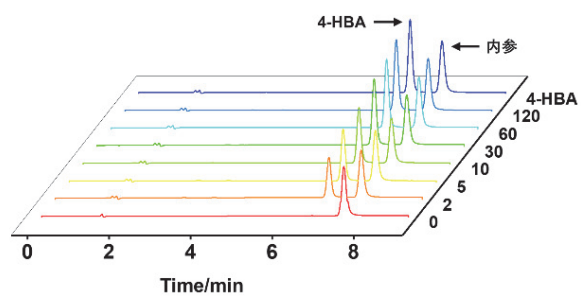

(d)

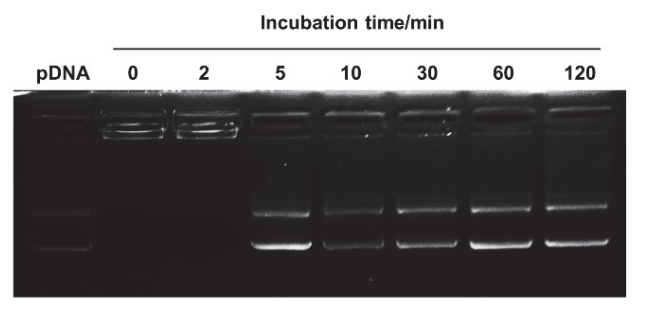

图 3 (a) pM-pBD 共聚物响应 ROS 实现电荷反转的分子机理. (b)在 $1 \mathrm{mmol} / \mathrm{L} \mathrm{H}_{2} \mathrm{O}_{2}$ 孵育不同时间 $(0 、 2 、 5 、 10 、 30 、 60 、 120 \mathrm{~min})$ 后释放出对羟

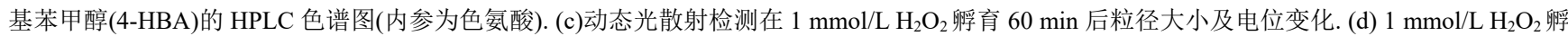
育后 $\mathrm{pM}-\mathrm{pBD} / \mathrm{pDNA}(\mathrm{N} / \mathrm{P}=3)$ 的凝胶阻滞电泳图

Figure 3 (a) The schematic diagram of pM-pBD copolymer producing polyacrylic acid by charge reversal in response to ROS. (b) HPLC chromatogram of 4-hydroxybenzyl alcohol (4-HBA) released after incubation in presence of $1 \mathrm{mmol} / \mathrm{L} \mathrm{H}_{2} \mathrm{O}_{2}$ for varied time $(0,2,5,10,30,60,120 \mathrm{~min})$ (internal reference: tryptophan). (c) The changes of particle size and $\zeta$ potential after incubation in presence of $1 \mathrm{mmol} / \mathrm{L} \mathrm{H}_{2} \mathrm{O}_{2}$ for 60 min was measured by dynamic light scattering. (d) Gel electrophoresis image of pM-pBD/pDNA (N/P=3) under incubation in presence of $1 \mathrm{mmol} / \mathrm{L} \mathrm{H}_{2} \mathrm{O}_{2}$ 
释放部分载荷基因使得表面电位降低. 为了进一步验证 载体响应活性氧后 pDNA 的释放行为, 进行了凝胶电泳 阻滞实验, 结果如图 $3 \mathrm{~d}$ 所示, 在 $1 \mathrm{mmol} / \mathrm{L} \mathrm{H}_{2} \mathrm{O}_{2}$ 处理 5 $\min$ 后, 在 pDNA 对应位置出现了相应的条带, 表明 ROS 能够促进载荷 pDNA 的释放; 相反, 在没有 $\mathrm{H}_{2} \mathrm{O}_{2}$ 的存在时, $\mathrm{pM}-\mathrm{pBD} / \mathrm{pDNA}$ 中 $\mathrm{pDNA}$ 被完全阻滞在上样 孔中. 以上结果证明了 ROS 刺激 $\mathrm{pM}-\mathrm{pBD}$ 发生级联断裂 导致电荷反转, 并能够促进 $\mathrm{pM}-\mathrm{pBD} / \mathrm{pDNA}$ 复合物解体 释放载荷 $\mathrm{pDNA}$.

\section{4 生物相容性分析}

良好的生物相容性是基因递送载体所必备的一个 关键性质. 为了研究 $\mathrm{pM}-\mathrm{pBD} / \mathrm{pDNA}$ 的生物相容性, 使 用 MTT 法测定不同 N/P 的纳米复合物对 HeLa 细胞的 细胞毒性. 结果如图 4a 所示, 不同 $\mathrm{N} / \mathrm{P}(0,0.5,1,2,3,4$, 6,8 和 16)情况下, HeLa 细胞存活率均大于 $80 \%$, 尤其是 $\mathrm{N} / \mathrm{P}=3$ 时, 载体浓度在 $0.1 \mathrm{mg} / \mathrm{mL}$ 时细胞存活率在 $90 \%$ 以上, 显示出较低的细胞毒性, 此结果说明了 $\mathrm{pM}-\mathrm{pBD} / \mathrm{pDNA}(\mathrm{N} / \mathrm{P}=3)$ 具有较好的生物相容性, 可以 用于后续的基因转染实验.

\section{5 抗坏血酸(AA)产生 ROS 实验}

据文献报道, 抗坏血酸及其衍生物在生理学浓度下 是抗氧化剂, 但在病理生理学浓度下具有促氧化作 用 ${ }^{[21]}$. 抗坏血酸能够被金属蛋白催化剂 $(\mathrm{Mn})$ 氧化为抗

(a)

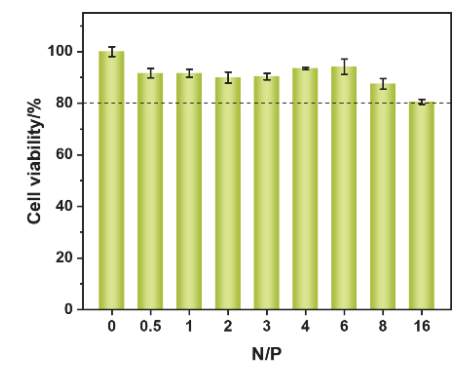

(c)

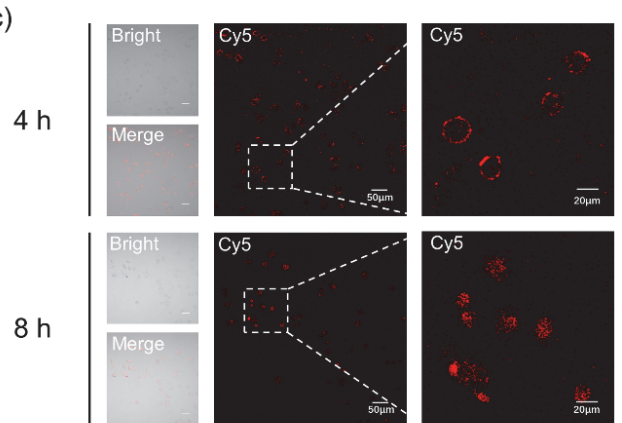

坏血酸自由基 $\left(\mathrm{AA}^{--}\right), \mathrm{AA}^{--}$将电子转移给氧分子, 形成 超氧自由基 $\left(\mathrm{O}_{2}{ }^{-}\right)$, 最终形成 $\mathrm{H}_{2} \mathrm{O}_{2}{ }^{[22]}$. 通过体外模拟体 液环境(含有 $10 \% \mathrm{FBS}$ 的 DMEM 培养基), 测定了不同 抗坏血酸的浓度下生成 $\mathrm{H}_{2} \mathrm{O}_{2}$ 的能力, 如图 $4 \mathrm{~b}$ 所示, 在 有抗坏血酸存在条件下, 在一定范围内, 溶液中 $\mathrm{H}_{2} \mathrm{O}_{2}$ 含 量随着孵育时间增加而不断增加, 并在 $240 \mathrm{~min}$ 左右达 到峰值, 随后逐渐降低, 该结果说明了, 抗坏血酸能够 促进产生 $\mathrm{H}_{2} \mathrm{O}_{2}$, 随后正常生理条件下生成的 ROS 会被 相关酶(过氧化氢酶等)消耗. 此外随着抗坏血酸浓度的 升高, 同比情况下会产生更多的 $\mathrm{H}_{2} \mathrm{O}_{2}$, 由此可知, 在病 理生理条件下, 产生的 $\mathrm{H}_{2} \mathrm{O}_{2}$ 不能被及时的调节时, 在一 定程度上能够增加 ROS 的浓度. 为了能够满足其在生 物体中的应用, 即能够产生足够的活性氧用于触发活性 氧敏感的基因载体释放载荷, 又能够在不影响细胞的活 性下提高转染效率, 利用 MTT 法检测了抗坏血酸对 HeLa 细胞的细胞毒性, 如图 S7 所示, 在抗坏血酸的浓 度在 $10 \mathrm{mmol} / \mathrm{L}$ 以下作用 $24 \mathrm{~h}$ 后，未发现明显的细胞毒 性(细胞存活率 $\geqslant 95 \%$ ), 说明 HeLa 细胞对抗坏血酸有一 定的耐受度, 单独的抗坏血酸不会影响细胞活性.

\section{6 细胞摄取的定性和定量分析实验结果}

利用激光共聚焦显微镜和流式细胞仪分别对 HeLa 细胞对 $\mathrm{pM}-\mathrm{pBD} / \mathrm{pDNA}$ 的摄取进行定性和定量分析. 首 先, 用 Cy5 对质粒 DNA (pDsRed2-N1)进行荧光标记制

(b)

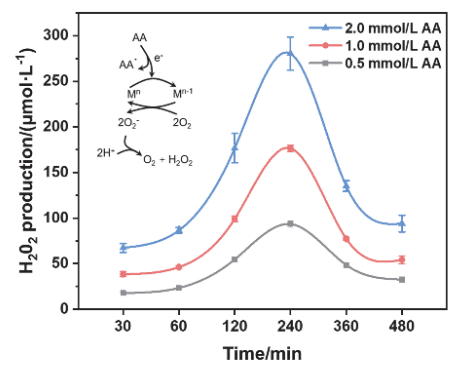

(d)
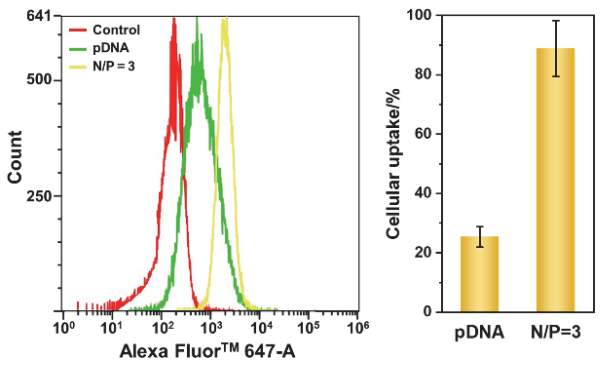

图 4 (a) $\mathrm{HeLa}$ 细胞在不同 $\mathrm{N} / \mathrm{P}$ 的 $\mathrm{pM}-\mathrm{pBD} / \mathrm{pDNA}$ 纳米复合物中捊育 $24 \mathrm{~h}$ 后的细胞存活率. (b)不同抗坏血酸浓度产 $\mathrm{H}_{2} \mathrm{O}_{2}$ 的能力(插图: 抗坏血酸 产生活性氧的机理). (c)激光共聚焦显微镜观察 $\mathrm{HeLa}$ 细胞对 $\mathrm{N} / \mathrm{P}=3$ 的 $\mathrm{pM}-\mathrm{pBD} / \mathrm{Cy} 5-\mathrm{pDNA}$ 纳米复合物在孵育 $4 \mathrm{~h}$ 及 $8 \mathrm{~h}$ 后的摄取. (d)流式细胞术 测定的 HeLa 细胞对 pDNA 和 $\mathrm{pM}-\mathrm{pBD} / \mathrm{Cy} 5-\mathrm{pDNA}$ 纳米复合物 $(\mathrm{N} / \mathrm{P}=3)$ 的摄取效率

Figure 4 (a) The cell viability of pM-pBD/pDNA nanocomplex at varied N/P ratios for $24 \mathrm{~h}$ in HeLa cell. (b) Production of $\mathrm{H}_{2} \mathrm{O}_{2}$ at varied concentrations of ascorbic acid (illustration: the mechanism of ascorbic acid producing reactive oxygen species). (c) Confocal laser scanning microscopy of HeLa cells incubated with pM-pBD/Cy5-pDNA $(\mathrm{N} / \mathrm{P}=3)$ nanocomplex for $4 \mathrm{~h}$ and $8 \mathrm{~h}$. (d) the uptake efficiency of pDNA and pM-pBD/Cy5-pDNA complexes $(\mathrm{N} / \mathrm{P}=3)$ in HeLa cells 
备 Cy5-pDNA，将其与 pM-pBD 复合制备 pM-pBD/ Cy5-pDNA 纳米复合物. 在与 HeLa 细胞孵育 $4 \mathrm{~h}$ 时(图 $4 \mathrm{c}$, Cy5 的红色苂光主要聚集在细胞膜周围, 说明纳米 复合物可能是通过内吞方式进入细胞, 此时的纳米复合 物主要存在于早期内涵体中 ${ }^{[23]}$; 随着孵育时间延长 $(8 \mathrm{~h}$ 时), 红色苂光从细胞膜周围逐渐扩散到整个细胞, 尤 其是细胞核. 此外, 流式细胞仪检测了在孵育 $8 \mathrm{~h}$ 后 HeLa 细胞内的 Cy5 荧光强度, 结果如图 4d 所示, 相比 于游离的 $\mathrm{pDNA}$ (摄取效率仅为 $25.4 \%$ ), HeLa 细胞对 N/P $=3$ 的纳米复合物具有极高的摄取效率 $(88.9 \%)$, 而且细 胞内荧光强度显著高于游离 $\mathrm{pDNA}$ 组, 说明 $\mathrm{pM}-\mathrm{pBD}$ 载 体能够有效地帮助 HeLa 细胞对 pDNA 的摄取, 从而能 够提高外源基因的转染效率.

\section{7 体外基因转染实验结果}

为了验证 $p M-p B D$ 载体的转染能力, 选择红色苂光 蛋白报告基因(pDsRed2-N1)质粒，与 $\mathrm{pM-pBD}$ 复合成纳 米粒子. 首先通过激光共聚焦显微镜对 HeLa 细胞中的 转染效果进行定性分析, 结果如图 5a 所示, 游离的 pDNA 组几乎没有红色苂光蛋白的表达. 相反, 载体组 中细胞的红色苂光强度明显增强, 而且随着 N/P 增加苂 光强度逐渐增强, 说明了 $\mathrm{pM}-\mathrm{pBD}$ 载体能够显著增强转 染效率. 此外, 通过流式细胞仪对转染效率进行定量分
析，如图 5b 所示，阳性对照组 $\mathrm{PEI}_{1000} / \mathrm{pDNA}(\mathrm{N} / \mathrm{P}=6.25$, 记为 $\mathrm{PEI} / \mathrm{pDNA})^{[24]}$ 组在 $\mathrm{HeLa}$ 细胞中的转染效率最高 $(51.0 \%), \mathrm{pDNA}$ 组几乎没有转染效果(仅为 $0.37 \%$ ). 然 而, $\mathrm{pM}-\mathrm{pBD} / \mathrm{pDNA}$ 的转染效率随着 $\mathrm{N} / \mathrm{P}$ 增加而增加, 相 比于 $\mathrm{N} / \mathrm{P}=1$ 组 $(2.6 \%), \mathrm{N} / \mathrm{P}=3$ 时的转染效率 $(25.9 \%)$ 显 著提高, 可能是由于该体系具有较好的粒径大小 $(99$ $\mathrm{nm})$ 和表面电位 $(+13.8 \mathrm{mV})$, 有利于细胞的内吞摄取, 从而提高了转染效率. 此外, $\mathrm{N} / \mathrm{P}=16$ 时的转染效率 (31.2\%)也较高, 但由于其具有更高的表面电位 $(+20.1$ $\mathrm{mV}$ ), 使得其具有更高的细胞毒性(图 4a), 从而不利于 后续应用。

此外, 为了验证抗环血酸的引入可以提高 pM-pBD/pDNA 对细胞的转染效率, 选择不同的细胞系 (HeLa、MCF-7 和 SGC-7901 细胞)利用流式细胞仪做定 量分析. 选择 $\mathrm{N} / \mathrm{P}=3$ 的纳米复合物作为实验对象, 结果 如图 5c 所示, 对于 HeLa 细胞, 加入抗坏血酸后, N/P=3 转染效率 $(38.8 \%)$ 是未加抗坏血酸组的 1.5 倍; 相比之下, $\mathrm{pDNA}$ 和 PEI/pDNA 组在加入抗坏血酸后转染效率几乎 没有变化 (图 S8a), 这说明抗坏血酸能够增强 $\mathrm{pM}-\mathrm{pBD} / \mathrm{pDNA}$ 的转染效率, 这是由于抗坏血酸的加入, 增加了 HeLa 细胞内的 ROS 水平, 促进 pM-pBD 的氧化 分解反应和电荷反转, 帮助 pDNA 的快速释放, 进而增

(a)
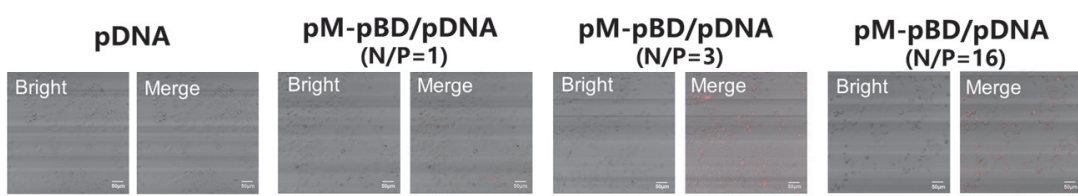

PEI/PDNA
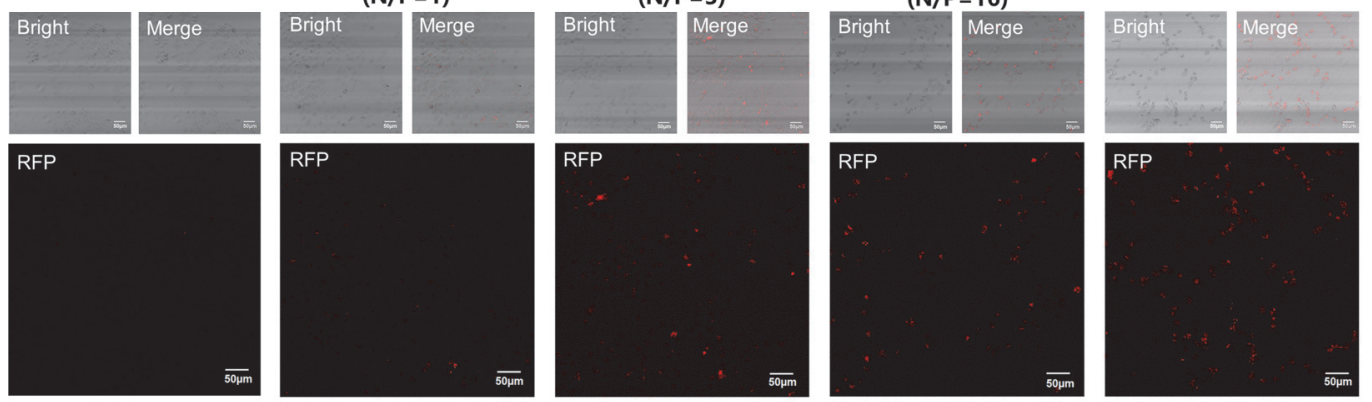

(b)

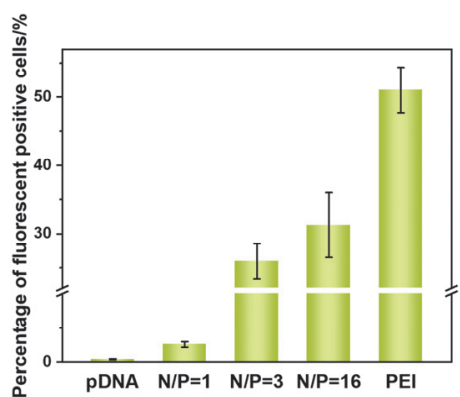

(c)

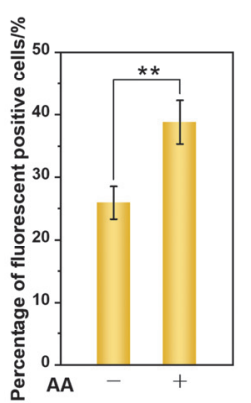

(d)

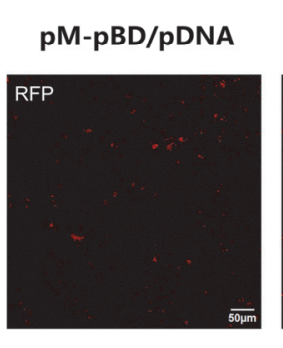

图 5 (a) $\mathrm{pDNA}$ 、不同 $\mathrm{N} / \mathrm{P}$ 的 $\mathrm{pM}-\mathrm{pBD} / \mathrm{pDNA}$ 和 $\mathrm{PEI} / \mathrm{pDNA}(\mathrm{N} / \mathrm{P}=6.25)$ 在 $\mathrm{HeLa}$ 细胞中转染后的激光共聚焦显微镜图. (b) $\mathrm{pDNA}$ 、不同 $\mathrm{N} / \mathrm{P}$ 的 $\mathrm{pM}-\mathrm{pBD} / \mathrm{pDNA}$ 和 $\mathrm{PEI} / \mathrm{pDNA}(\mathrm{N} / \mathrm{P}=6.25)$ 在 $\mathrm{HeLa}$ 细胞中的转染效率. (c)有/无抗坏血酸存在时 $\mathrm{pM}-\mathrm{pBD} / \mathrm{pDNA}$ 复合物 $(\mathrm{N} / \mathrm{P}=3)$ 在 $\mathrm{HeLa}$ 细胞中的转 染效率. (d)有/无抗坏血酸存在时 $\mathrm{pM}-\mathrm{pBD} / \mathrm{pDNA}$ 复合物 $(\mathrm{N} / \mathrm{P}=3)$ 在 $\mathrm{HeLa}$ 细胞中转染后的激光共聚焦显微镜图

Figure 5 (a) Confocal laser scanning microscopy of HeLa cells against $\mathrm{pDNA}, \mathrm{pM}-\mathrm{pBD} / \mathrm{pDNA}$ with varied N/P ratios and PEI/pDNA (N/P=6.25). (b) The transfection efficiency of pDNA, pM-pBD/pDNA with varied N/P ratios and PEI/pDNA (N/P=6.25) in HeLa cells. (c) The transfection efficiency of $\mathrm{pM}-\mathrm{pBD} / \mathrm{pDNA}$ complex $(\mathrm{N} / \mathrm{P}=3)$ with or without ascorbic acid (AA) in HeLa cells. (d) Confocal laser scanning microscopy of the transfection of $\mathrm{pM}-\mathrm{pBD} / \mathrm{pDNA}$ complex $(\mathrm{N} / \mathrm{P}=3)$ with or without ascorbic acid in HeLa cells 
加转染效率. 于此同时, 在 MCF-7 和 SGC-7901 细胞中 得到了类似的结果(图 $\mathrm{S} 8 \mathrm{~b}$ ). 此外, 通过激光共聚焦可 视化地观察了 $\mathrm{pM}-\mathrm{pBD} / \mathrm{pDNA}$ 在有/无抗坏血酸存在时 的红色菼光蛋白质粒的转染情况, 结果如图 5d 所示, 在 加入 $1 \mathrm{mmol} / \mathrm{L}$ 的抗坏血酸后, HeLa 细胞内的红色苂光 显著增强, 进一步证明了抗坏血酸的引入能够通过增加 ROS 水平, 提高 $\mathrm{pM}-\mathrm{pBD} / \mathrm{pDNA}$ 复合物的转染效率.

\section{3 结论}

本工作以 MPC、 BD 为原料通过 RAFT 法合成了能 够响应 ROS 选择性断裂的阳离子聚合物 pM-pBD. 通过 $\mathrm{pM}-\mathrm{pBD}$ 与基因复合制备了活性氧响应性的基因载体复 合物, 并显示出较好的粒径分布和结构稳定性; 实验结 果验证了 pM-pBD 能够响应 ROS 发生电荷反转(正电荷 向负电荷的转换), 为帮助载荷卸载提供了实验基础;

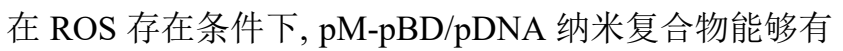
效地释放载荷 $\mathrm{pDNA}$, 从而提高了载体的基因转染效率. 细胞层面结果表明 $\mathrm{pM}-\mathrm{pBD} / \mathrm{pDNA}$ 几乎没有细胞毒性, 能够显著提高细胞的摄取效率和转染效率, 此外, 抗坏 血酸的存在能有效地提高 $\mathrm{pM}-\mathrm{pBD} / \mathrm{pDNA}$ 的转染效率. 因此，合成和制备的 $\mathrm{pM}-\mathrm{pBD}$ 基因平台为开发安全、高 效、可控释放的基因递送系统提供了新的思路.

\section{4 实验部分}

\section{1 实验试剂与仪器}

本实验所使用的试剂及仪器详细信息参见支持信 息.

\section{2 pM-pBD 的合成}

聚阳离子共聚物 pM-pBD 按照图 1 所示的合成路线 进行合成. 首先, 将 DEAEA (1.370 $\mathrm{g}, 8 \mathrm{mmol})$ 与 $\mathrm{B}$ (3.564 g, $12 \mathrm{mmol}$ )溶解于 $N, N-$ 二甲基甲酰胺 $(20 \mathrm{~mL})$ 中, 在室温下持续搅拌 $24 \mathrm{~h}$, 将反应后的溶液缓慢滴加到冰 乙醚中, 收集沉淀并用冰乙醚洗 2 次, 干燥后得到白色 固体 BD (3.184 g, 产率: 84.9\%). 其次, 将 MPC (1.181 $\mathrm{g}, 4 \mathrm{mmol})$ 、氘基戊酸二硫代苯甲酸酯 $(0.279 \mathrm{~g}, 0.1$ $\mathrm{mmol})$ 和偶氮二氰基戊酸 $(0.006 \mathrm{~g}, 0.02 \mathrm{mmol})$ 添加到 2

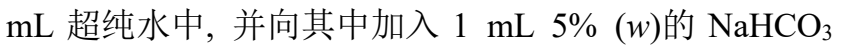
溶液, 置于冰上搅拌至溶解, 之后通氮气 $30 \mathrm{~min}$ 使溶液 脱氧. 然后将其转移至 $70{ }^{\circ} \mathrm{C}$ 的油浴中, 搅拌反应 $4 \mathrm{~h}$. 冷却后, 导入透析袋 $\left(M_{\mathrm{w}}\right.$ : 3000) 中用去离子水透析 3 $\mathrm{d}$ (每 $8 \mathrm{~h}$ 换一次透析液). 通过冷冻干燥回收获得粉色固 体 pMPC. 最后, 将合成的 BD (7.766 g, $20 \mathrm{mmol})$ 、 pMPC $(1.827 \mathrm{~g}, 0.2 \mathrm{mmol})$ 和偶氮二氭基戊酸 $(0.014 \mathrm{mg}$, $0.05 \mathrm{mmol}$ )添加到 $20 \mathrm{~mL}$ 超纯水中, 反应过程同 $\mathrm{pMPC}$ 的合成, 并通过冷冻干燥回收获得淡粉色固体 pM-pBD. 利用 Q-TOF Micro 高分辨质谱仪对化合物 BD 的分子量 进行表征. 利用 Bruker AVANCE III 500 核磁共振氢谱
对合成的化合物 BD, pMPC 及 pM-pBD 进行结构表征, $\mathrm{CDCl}_{3}$ 和 $\mathrm{D}_{2} \mathrm{O}$ 为溶剂, TMS 作内标 $(\delta 0.00)$, 通过凝胶渗 透色谱(GPC)测定 $\mathrm{pM}-\mathrm{pBD}$ 共聚物的分子量和均一性, 色谱柱: PL aquagel-OH (MIXD-H, $8 \mu \mathrm{m}$ ), 流动相: 500 $\mathrm{mmol} / \mathrm{L} \mathrm{NaCl}$ 溶液，温度: $25{ }^{\circ} \mathrm{C}$, 流速: $1.0 \mathrm{~mL} / \mathrm{min}$.

\section{$4.3 \mathrm{pM}-\mathrm{pBD} / \mathrm{pDNA}$ 纳米复合物的制备及表征}

按照不同 N/P (N/P: 载体 $\mathrm{pM}-\mathrm{pBD}$ 与 $\mathrm{pDNA}$ 的质量 比)制备 $\mathrm{pM}-\mathrm{pBD} / \mathrm{pDNA}$ 纳米复合物. 首先使用低温的 4-差欮乙基哌嗪乙磺酸(HEPES)缓冲液 $(10 \mathrm{mmol} / \mathrm{L}, \mathrm{pH}=$ 7.4)将载体充分溶解, 然后逐滴加到 $\mathrm{pDNA}$ 溶液中, 浴 旋震荡混匀. 整个操作过程要在低温条件下进行以确保 pDNA 的稳定性.

使用 Zetasizer Nano ZS90 纳米粒度仪测量不同 N/P $(1 、 2 、 3 、 4 、 6 、 8 、 16)$ 纳米复合物的粒径和电位及多 分散性指数.

使用 TECNAI Spirit $(120 \mathrm{kV})$ 透射电子显微镜观察 纳米复合物的形态. 首先, 制备 $\mathrm{N} / \mathrm{P}=3$ 的 $\mathrm{pM}-\mathrm{pBD} /$ pDNA 纳米复合物, 随后将铜网浸入到纳米复合物溶 液-醋酸双氧铀混合溶液中(体积比为 $1: 1$ ), 孵育 10 $\min$, 之后取出铜网在空气中静置至其表面完全干燥.

使用 DYY-8C 凝胶电泳仪对不同 N/P (0、0.5、1、 $1.5 、 2 、 3 、 4 、 6 、 8 、 16)$ 纳米复合物进行凝胶电泳分析. 电泳缓冲液为 $1 \times \mathrm{TAE}$, 电泳凝胶为 $1.0 \%$ 琼脂糖凝胶 $($ 含 $0.1 \mu \mathrm{g} / \mathrm{mL}$ Gel stain), 在上样前每孔加入适量 Loading Buffer. 电泳条件: 电压 $110 \mathrm{~V}$ ，电泳时间 $40 \mathrm{~min}$. 电泳 结束后用凝胶成像仪系统观察并拍照.

为了验证不同 $\mathrm{N} / \mathrm{P}$ 值下纳米复合物在血清中的稳定 性，使用 Zetasizer Nano ZS90 纳米粒度仪测量不同 N/P 值 $(1 、 2 、 3 、 4 、 6 、 8 、 16)$ 的纳米复合物在 $37{ }^{\circ} \mathrm{C}$ 下与 含有 $10 \%$ 血清的 HEPES 缓冲液孵育 $2 \mathrm{~h} 、 4 \mathrm{~h}$ 和 $6 \mathrm{~h}$ 后 的粒径变化情况.

为了检测纳米复合物对 $\mathrm{pDNA}$ 的包覆能力, 将 N/P $=3$ 的 $\mathrm{pM}-\mathrm{pBD} / \mathrm{pDNA}$ 复合物(含 $1 \mu \mathrm{g} \mathrm{pDNA}$ )加入不同 浓度肝素溶液(终浓度 $0 、 1 、 5 、 20 、 80 \mathrm{mg} / \mathrm{mL}$ ), 在 $37{ }^{\circ} \mathrm{C}$ 水浴 $2 \mathrm{~h}$, 进行琼脂糖凝胶电泳分析. 电泳条件如上.

\section{4 pM-pBD 及 pM-pBD/pDNA 纳米复合物响应活性 氧测定}

使用 Agilent 1260 infinity 高效液相色谱仪对 pM-pBD 响应活性氧前后的结构变化进行分析. 在 pM-pBD $(0.3 \mathrm{mg} / \mathrm{mL})$ 的溶液中加入 $\mathrm{H}_{2} \mathrm{O}_{2}(1 \mathrm{mmol} / \mathrm{L})$, $37{ }^{\circ} \mathrm{C}$ 孵育, 在特定的时间点取样 $20 \mu \mathrm{L}$ 溶液并进行 HPLC 分析, 色谱柱: C18 色谱柱(Poroshell 120, $2.7 \mu \mathrm{m}$, $4.6 \mathrm{~mm} \times 100 \mathrm{~mm}$ ), 流动相: $10 \%$ 甲醇水溶液，流速： 1.0 $\mathrm{mL} / \mathrm{min}$ ，检测波长: $280 \mathrm{~nm}$ ，内标物：色氨酸.

在含 $\mathrm{pM}-\mathrm{pBD} / \mathrm{pDNA}$ 纳米复合物的缓冲液中加入 $\mathrm{H}_{2} \mathrm{O}_{2}(1 \mathrm{mmol} / \mathrm{L})$, 随后在不同时间点测定复合物的粒径 和电位及多分散性指数. 
通过凝胶阻滞电泳检测纳米复合物在氧化条件下 的 DNA 释放. 将 $\mathrm{N} / \mathrm{P}=3$ 的复合物在 $1 \mathrm{mmol} / \mathrm{L} \mathrm{H}_{2} \mathrm{O}_{2}$ 中 于 $37{ }^{\circ} \mathrm{C}$ 孵育. 在特定的时间点取样, 然后如上所述方 法进行电泳.

\section{5 细胞毒性实验}

使用 MTT 法对 $\mathrm{pM}-\mathrm{pBD} / \mathrm{pDNA}$ 复合物的细胞毒性 进行分析. 以 $1 \times 10^{5}$ 个 $/ \mathrm{mL}$ 的密度将 HeLa 细胞接种于 96 孔板 $(100 \mu \mathrm{L})$. 在 $37{ }^{\circ} \mathrm{C} 、 5 \% \mathrm{CO}_{2}$ 的细胞培养箱中孵 育 $24 \mathrm{~h}$. 弃去旧培养基, 然后每孔加入 $100 \mu \mathrm{L}$ 含有不同 浓度的 $\mathrm{pM}-\mathrm{pBD} / \mathrm{pDNA}$ 纳米复合物的培养基(pDNA 浓 度均为 $5 \mathrm{ng} / \mu \mathrm{L}$ ), 不加药物组为正常对照, 不含细胞的 对照孔为空白对照, 培养 $24 \mathrm{~h}$ 后, 检测细胞的存活率. 检测时弃掉细胞的培养液, 每孔加入 $100 \mu \mathrm{L}$ MTT 溶液 $(0.5 \mathrm{mg} / \mathrm{mL})$ 继续培养 $4 \mathrm{~h}$, 小心吸去培养液并加入二甲 基亚砜(100 $\mu \mathrm{L} /$ 孔)溶解蓝紫色结晶物, 稍微振荡, 使用 酶标仪测定在 $570 \mathrm{~nm}$ 处的吸光度值(参比波长 $630 \mathrm{~nm}$ ). 细胞存活率的计算公式: 细胞存活率 $(\%)=[(\mathrm{OD}$ 样品孔 $\mathrm{OD}$ 空白组 $) /(\mathrm{OD}$ 对照孔 $-O D$ 空白组 $)] \times 100 \%$.

\section{6 抗坏血酸产生 ROS 能力测定}

将终浓度为 $0.5 、 1.0$ 和 $2.0 \mathrm{mmol} / \mathrm{L}$ 的抗坏血酸(AA) 添加到含有 $10 \% \mathrm{FBS}$ 的 DMEM 培养基中, 并在室温下 孵育. 在预定的时间点取出 $10 \mathrm{~mL}$ 溶液, 向其中添加 1000 单位的过氧化氢酶溶液 $(200 \mu \mathrm{L})$, 随后利用溶解氧 (D.O.)仪测量氧气的产量.

\section{7 细胞摄取定性和定量实验}

使用激光共聚焦显微镜可视化地观察 HeLa 细胞对 pDNA 和 pM-pBD/pDNA 的摄取情况, 首先将 HeLa 细 胞以 $1 \times 10^{5}$ 个 $/ \mathrm{mL}$ 的密度接种于 $35 \mathrm{~mm}$ 激光共聚焦专 用血中, 放入 $37{ }^{\circ} \mathrm{C} 、 5 \% \mathrm{CO}_{2}$ 的细胞培养箱中孵育 $24 \mathrm{~h}$. 随后每孔加入 $45 \mu \mathrm{L}$ pDNA 或者 $\mathrm{N} / \mathrm{P}=3$ 的 pM-pBD/Cy5-pDNA(用 Cy5 标记的 pDNA)纳米复合物 (pDNA 的浓度均为 $2 \mathrm{ng} / \mu \mathrm{L}$ ), 放入细胞培养箱, 继续孵 育 $4 \mathrm{~h}$ 或 $8 \mathrm{~h}$, 用 PBS 清洗 2 次后, 使用 FV-1000 激光共 聚焦显微镜观察其摄取情况(Cy5 的激发波长为 635 $\mathrm{nm})$.

使用流式细胞仪定量测定 HeLa 细胞对 $\mathrm{pM}-\mathrm{pBD} / \mathrm{pDNA}$ 的摄取情况, 首先, 将 HeLa 细胞以 $1 \times 10^{5}$ 个 $/ \mathrm{mL}$ 的密度接种于 6 孔板中, 放入 $37{ }^{\circ} \mathrm{C} 、 5 \%$ $\mathrm{CO}_{2}$ 的细胞培养箱中孵育 $24 \mathrm{~h}$, 随后每孔加入 $45 \mu \mathrm{L}$ pDNA 或者 $\mathrm{N} / \mathrm{P}=3$ 的 $\mathrm{pM}-\mathrm{pBD} / \mathrm{Cy} 5-\mathrm{pDNA}$ 纳米复合物 (pDNA 的浓度均为 $2 \mathrm{ng} / \mu \mathrm{L}$ ), 放入细胞培养箱, 继续孵 育 $8 \mathrm{~h}$, 用 PBS 清洗 2 次, 胰蛋白酶消化收集细胞, 随后 用于流式细胞仪上机检测(Cy5 的激发波长为 $635 \mathrm{~nm}$ ).

\section{8 细胞转染效率定性和定量分析}

使用激光共聚焦显微镜可视化地观察 pDNA、 $\mathrm{pM}-\mathrm{pBD} / \mathrm{pDNA}$ 和 $\mathrm{PEI} / \mathrm{pDNA}(\mathrm{N} / \mathrm{P}=6.25)$ 在 $\mathrm{HeLa}$ 细胞 中的转染情况, 以及在有/无抗环血酸存在下的转染情
况. 首先将 $\mathrm{HeLa}$ 细胞以 $1 \times 10^{5}$ 个 $/ \mathrm{mL}$ 的密度接种于 35 $\mathrm{mm}$ 激光共聚焦专用血中, 放入 $37{ }^{\circ} \mathrm{C} 、 5 \% \mathrm{CO}_{2}$ 的细胞 培养箱中孵育 $24 \mathrm{~h}$. 随后每孔加入 $45 \mu \mathrm{L} \mathrm{pDNA}, \mathrm{N} / \mathrm{P}=$ $1 、 3$ 或 16 的 pM-pBD/pDNA 和 PEI/pDNA(阳性对照) 纳米复合物 (pDNA 的浓度均为 $2 \mathrm{ng} / \mu \mathrm{L}$ ), 放入细胞培养 箱, 继续孵育 $6 \mathrm{~h}$, 更换新鲜培养基继续培养 $42 \mathrm{~h}$. 抗坏 血酸处理组需要在更换新鲜培养基 $2 \mathrm{~h}$ 后加入抗坏血酸 (终浓度为 $1 \mathrm{mmol} / \mathrm{L}$ ), 继续培养 $40 \mathrm{~h}$. 用 PBS 清洗 2 次 后使用 FV-1000 激光共聚焦显微镜观察转染情况(RFP 的激发波长为 $559 \mathrm{~nm}$ ).

使用流式细胞仪定量测定 $\mathrm{pM}-\mathrm{pBD} / \mathrm{pDNA}$ 在有/无 抗坏血酸存在时，对不同细胞 (HeLa、MCF-7 和 SGC-7901)的转染情况. 细胞以 $1 \times 10^{5}$ 个 $/ \mathrm{mL}$ 的密度接 种于 6 孔板中, 放入 $37{ }^{\circ} \mathrm{C} 、 5 \% \mathrm{CO}_{2}$ 的细胞培养箱中孵 育 $24 \mathrm{~h} .24 \mathrm{~h}$ 后弃掉原培养基，随后每孔加入 $45 \mu \mathrm{L}$ $\mathrm{pDNA}, \mathrm{N} / \mathrm{P}=1 、 3$ 或 16 的 $\mathrm{pM}-\mathrm{pBD} / \mathrm{pDNA}$ 和 PEI/pDNA 纳米复合物 (pDNA 的浓度均为 $2 \mathrm{ng} / \mu \mathrm{L}$ ), 放入细胞培养 箱, 继续孵育 $6 \mathrm{~h}$, 更换新鲜培养基继续培养 $42 \mathrm{~h}$. 抗坏 血酸处理组需要在更换新鲜培养基 $2 \mathrm{~h}$ 后加入抗坏血酸 (终浓度为 $1 \mathrm{mmol} / \mathrm{L}$ ), 继续培养 $40 \mathrm{~h}$. 用 PBS 清洗 2 次, 胰蛋白酶消化收集细胞, 随后用于流式细胞仪上机进行 定量检测(RFP 的激发波长为 $561 \mathrm{~nm}$ ).

\section{9 统计学分析}

统计分析采用 OriginPro(9.5)软件进行单因素方差 分析(ANOVA), 数据以 mean \pm S.D.表示, 除特殊说明外 所有实验均进行三次重复, $p<0.05$ 认为有显著差异(采 用“*”表示); $p<0.01$ 认为有极显著差异(采用“***表示).

\section{References}

[1] Shen, Y.; Hu, G. X.; Zhang, H. X.; Qi, L. L.; Luo, C. C. Acta Chim. Sinica 2013, 71, 323 (in Chinese). (沈银, 胡桂香, 张华星, 齐莉 莉, 骆成才, 化学学报, 2013, 71, 323.)

[2] Hirko, A.; Tang, F. X.; Hughes, J. A. Curr. Med. Chem. 2003, 10, 1185.

[3] Nemunaitis, J.; Tong, A. W.; Nemunaitis, M.; Senzer, N.; Phadke, A. P.; Bedell, C.; Adams, N.; Zhang, Y. A.; Maples, P. B.; Chen, S.; Pappen, B.; Burke, J.; Ichimaru, D.; Urata, Y.; Fujiwara, T. Mol. Ther. 2010, 18, 429

[4] Farmer, Z. L.; Kim, E. S.; Carrizosa, D. R. Oral. Maxil. Surg. Clin. 2018, 31, 117.

[5] Rai, M. F.; Sandell, L. J. J. Am. Acad. Orthop. Sur. 2015, 23, 10.

[6] Wirth, T.; Yla-Herttuala, S. Biomedicines 2014, $2,2$.

[7] Lohr, M. Z. Gastroenterol. 2006, 44, 4.

[8] Lin, G. M.; Li, L.; Panwar, N.; Wang, J.; Tjin, S. C.; Wang, X. M.; Yong, K. T. Coordin. Chem. Rev. 2018, 374, 133.

[9] Prokofjeva, M. M.; Proshkina, G. M.; Lebedev, T. D.; Shulgin, A. A.; Spirin, P. V.; Prassolov, V. S.; Deyev, S. M. Biochimie. 2017, 142, 226.

[10] Wang, Q.; Zhang, L.; Chen, S. J. China Basic Sci. 2017, 19, 21 (in Chinese). (王嫱, 张琳, 陈赛娟, 中国基础科学, 2017, 19, 21.)

[11] Li, S. D.; Huang, L. J. Control. Release 2007, 123, 3.

[12] Liu, L.; Yang, J. Y.; Men, K.; He, Z. Y.; Luo, M.; Qian, Z. Y.; Wei, X. W.; Wei, Y. Q. Hum. Gene. Ther. 2018, 29, 110.

[13] Luo, D.; Saltzman, W. M. Nat. Biotechnol. 2000, 18, 33.

[14] Zhou, F.; Wu, C.; Han, F. X.; Zhao, Y. H.; Yuan, X. Y. J. Control. Release 2015, 213, E32.

[15] Srinivas, U. S.; Tan, B. W. Q.; Vellayappan, B. A.; Jeyasekharan, A. D. Redox Biol. 2019, 25, 101084.

[16] Zhao, W. J.; Qiao, Z. Y.; Duan, Z. Y.; Wang, H. Acta Chim. Sinica 
2016, 74, 234 (in Chinese). (赵文静, 乔增荣, 段中余, 王浩, 化学 学报, 2016, 74, 234.)

[17] Zhang, L. W.; Chen, Q. X.; Wang, J. Y. Acta Chim. Sinica 2020, 78, 642 (in Chinese). (张留伟, 陈麒先, 王静云, 化学学报, 2020, 78, 642.)

[18] Stone, J. R.; Yang, S. Antioxid Redox Sign. 2006, 8, 243.

[19] Zhang, J. H.; Wang, W. J.; Zhang, J.; Xiao, Y. P.; Liu, Y. H.; Yu, X. Q. Eur. J. Med. Chem. 2019, 182, 111666.

[20] Shim, M. S.; Xia, Y. N. Angew. Chem. Int. Ed. 2013, 52, 2926.
[21] Chen, Q.; Espey, M. G.; Sun, A. Y.; Pooput, C.; Kirk, K. L.; Krishna, M. C.; Khosh, D. B.; Drisko, J.; Levine, M. Biochemistry 2008, 105, 11105.

[22] Li, J. J.; Ke, W. D.; Wang, L.; Huang, M. M.; Yin, W.; Zhang, P.; Chen, Q. X.; Ge, Z. S. J. Control. Release 2016, 225, 64.

[23] Zhang, L. W.; Qian, M.; Cui, H. Y.; Zeng, S.; Wang, J. Y.; Chen, Q. X. ACS Appl. Mater. Interfaces 2021, 13, 6053.

[24] Wang, J. Y.; Dou, B. R.; Rao, Y. M. Mat. Sci. Eng. C-Mater. 2014, 34,98 .

(Cheng, B.) 